\title{
Expression and Amplification of Chirality in Two-Dimensional Molecular Crystals
}

\author{
Karl-Heinz Ernst* \\ Dedicated to Professor Jack D. Dunitz on the occasion of his $85^{\text {th }}$ birthday
}

\begin{abstract}
Chirality can be bestowed onto achiral surfaces by adsorption of chiral molecules. This offers a good opportunity to study two-dimensional chiral crystallization phenomena, like lateral resolution of enantiomers or the transfer of handedness from single molecules into mesoscopic ensembles with high resolution by scanning probe microscopy. Induction of homochirality on surfaces via cooperatively amplified interactions in molecular monolayers is a new phenomenon of supramolecular surface chirality. Prochiral molecules will turn into either handedness upon adsorption, but doping with intrinsically chiral molecules breaks this symmetry and induces homochirality. A similar effect is induced by a small enantiomeric excess. The excess molecules provide the chiral bias that becomes amplified into single lattice chirality.
\end{abstract}

Keywords: Chirality · Conglomerates $\cdot$ Crystallization $\cdot$ Enantiomorphism $\cdot$ Scanning tunneling microscopy

\section{Introduction}

In his famous experiment in 1848 Pasteur separated left- and right-handed ammonium sodium tartrate crystals manually and observed opposite optical activity for their aqueous solutions. ${ }^{[1]}$ His insight that the origin of chirality is based on molecular structure laid the foundation of modern structural organic chemistry. Two important details of his experiment actually allowed the manual separation: i) Handedness was transferred from the molecular structure to the macro-

${ }^{\star}$ Correspondence: PD Dr. K.-H. Ernst

Molecular Surface Science

Laboratory for Nanoscale Materials Science

Empa - Swiss Federal Laboratories for Materials

Testing and Research

Überlandstrasse 125

$\mathrm{CH}-8600$ Dübendorf

and University of Zürich

Institute of Organic Chemistry

Winterthurerstrasse 190

$\mathrm{CH}-8057$ Zürich

Tel.: +41448234363

Fax: +41448234034

E-mail: Karl.Heinz.Ernst@empa.ch scopic shape of the crystal (hemihedrism), and ii) the racemate precipitated into a conglomerate of homochiral crystals. The underlying mechanisms of both processes are still poorly understood. We are neither able to predict the shape of a crystal from molecular structure ${ }^{[2]}$ nor do we know why less than ten percent of all racemates crystallize into conglomerates. ${ }^{[3,4]}$ One reason for the difficulty to understand and investigate these processes is their cooperative nature. Extremely small structural influences govern the macroscopic result when they become amplified by many cooperating units. Cooperativity among different homochiral biomolecules is also of fundamental importance in life. ${ }^{[5]}$ Furthermore, the transfer of chirality into mesoscopic structures $-\mathrm{a}$ very important issue in liquid crystal science - is difficult to predict in three dimensions. More tractable and easier understood are two-dimensional (2D) crystals formed at surfaces, in particular because of the possibility to study these processes at the molecular level by exploitation of scanning tunneling microscopy (STM). Different aspects of chirality in two-dimensional molecular lattices self-assembled on $C_{3 \mathrm{v}^{-}}$and $C_{2 \mathrm{v}}$-symmetric single-crystalline copper surfaces are reviewed. The examples include monolayers of enantiopure and racemic heptahelicene $\left(\mathrm{C}_{30} \mathrm{H}_{18}\right)$, a helically shaped aromatic hydrocarbon on $\mathrm{Cu}(111)$, racemic tartaric acid on $\mathrm{Cu}(110)$ as well as chirally doped meso-tartaric acid and succinic acid on $\mathrm{Cu}(110)$.

\section{Methods and Materials}

All adsorbate systems have been investigated under ultra-high vacuum (UHV) conditions $\left(p \approx 10^{-8} \mathrm{~Pa}\right)$. The organic compounds were evaporated from Knudsen cells in vacuo onto the copper crystal surfaces at room temperature. The polished $\mathrm{Cu}$ crystal surfaces (MaTecK) were cleaned via argon ion bombardment and were subsequently annealed at $950 \mathrm{~K}$ for one minute. Cleanliness and quality of the surfaces and the surface coverage of the adsorbate systems were determined via Xray photoelectron spectroscopy (XPS), low energy electron diffraction (LEED), temperature programmed desorption (TPD) and STM. TPD curves were acquired with a heating rate of $4 \mathrm{~K} \mathrm{~s}^{-1}$ using a quadrupole mass spectrometer as detector. Synthesis and enantiomeric separation ( $e e>99.9 \%$ ) of heptahelicene $([7] \mathrm{H})$ was performed as described previously. ${ }^{[6]}$ The absolute configuration was assigned to a high level of confidence by comparison of experimental and calculated VCD spectra. ${ }^{[7]}$ $\mathrm{X}$-ray photoelectron diffraction studies (XPD) of $(M)$-[7]H on the stepped $\mathrm{Cu}(332)$ surface also confirmed this assignment. ${ }^{[8]}$ Tartaric acid $(R, S-, R, R-, S, S$ - \& racemate) and succinic acid were purchased from Aldrich and Merck with purities better than 99\%. STM images were acquired in constant-current mode with the sample slowly cooled to $50 \mathrm{~K}$. Molecular modeling calculations (MMC) were performed using 
the AMBER force field of the Hyperchem 7 package.

\section{Results and Discussion}

\subsection{Transfer of Handedness from Single Molecules into 2D Crystals}

Pure enantiomers at surfaces form in most cases 2D enantiomorphous structures in close-packed monolayers. ${ }^{[9]}$ That is, chirality is transferred from the single molecule into a long-range chiral motif at the surface. The created motifs are mirror images for the opposite enantiomers. One example are the pure [7] $\mathrm{H}$ enantiomers on $\mathrm{Cu}(111) .{ }^{[10]}$ Close to monolayer saturation, handed pinwheel structures are observed via STM (Fig 1a,b). The (M)-[7]H-pinwheels have the opposite handedness to the $(P)$-[7] $\mathrm{H}$-pinwheels. In addition, at full monolayer coverage 'three-molecule-cloverleaf' units show opposite tilt angles with respect to the adsorbate lattice unit cell (Fig 1c,d). In all cases, the adsorbate lattice is aligned in an oblique angle to the underlying substrate lattice. Especially this latter mode of expression of chirality in ordered adsorbate systems is a common phenomenon. ${ }^{[9,11]}$ Molecular modeling calculations (MMC) reveal that these structures are governed by steric constraints. The lowest-energy structure for the close-packed monolayer (Fig. $1 \mathrm{c}, \mathrm{d})$ delivers identical adsorption sites for all molecules of the unit cell. Their respective azimuthal orientations, however, are different and depend strongly on the sign of helicity of the molecules, so that opposite lattice enantiomorphism is observed for the enantiomers. Assuming that the brightest features of the STM images reflect the off-centered topmost part of the molecular helix, the MMC-structure in Fig. 1e agrees well with the experimentally observed STM structure in Fig. 1c. ${ }^{[10]}$ Similar to the frustrated lattice structures observed for crystalline polymers of single helicity, e.g. isotactic poly(propylene), ${ }^{[12]}$ not all helices can be aligned 'in phase'. This shows that, although the packing is mediated by the $\mathrm{Cu}$ substrate grid, the 'one-pitch' [7]H helices follow the same sterically controlled packing rules as extended helical molecules in 3D crystals. [7] $\mathrm{H}$ on $\mathrm{Cu}(111)$ is therefore an example for short-range sterically controlled supramolecular self-assembly mediated by the surface lattice.

\subsection{Racemic Crystals versus Conglomerates}

It has been predicted that two-dimensional enantiomeric resolution on a surface should occur more easily than in 3D crystals. Due to confinement in the plane certain symmetry elements, e.g. the center of inversion or the glide plane parallel to the surface, are precluded and enhanced chiral
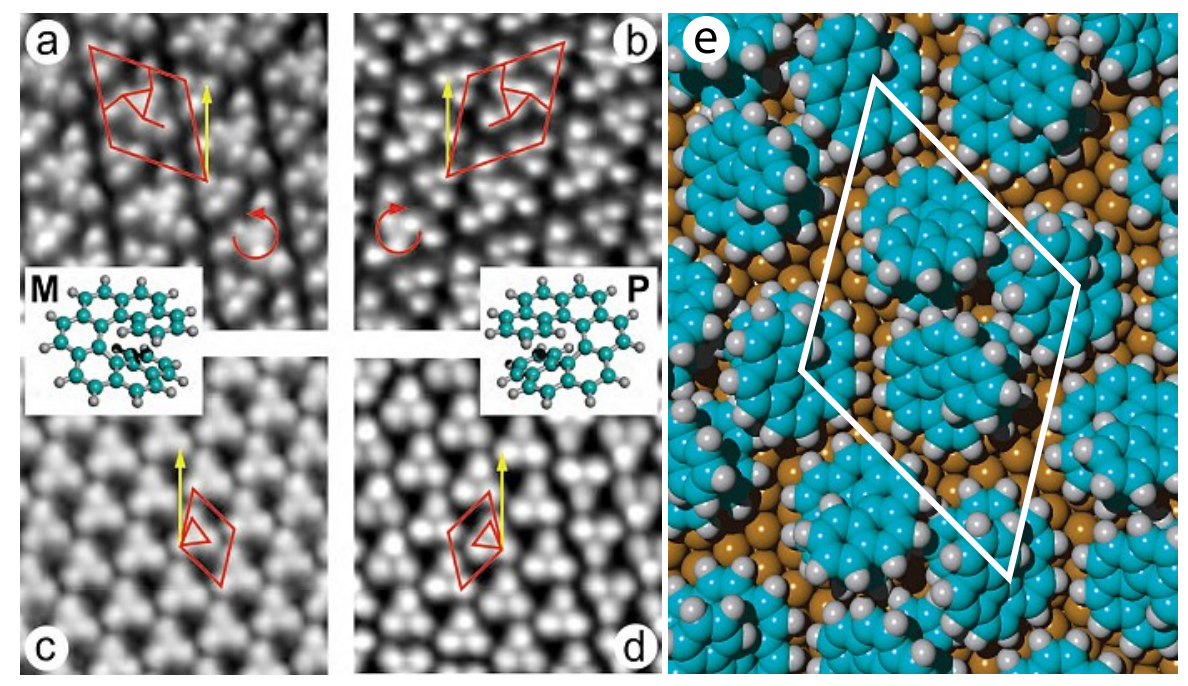

Fig. 1. (a-d) High-resolution STM images $(10 \mathrm{~nm} \times 10 \mathrm{~nm})$ of $(M)$ - and $(P)-[7] \mathrm{H}$ structures. The respective adlattices (red unit cells) have opposite tilt angles with respect to the substrate lattice (yellow arrow). (M)-[7] H forms at $91 \%$ of the saturated monolayer clockwise rotated pinwheels (a), while counterclockwise rotated pinwheels are observed via STM for $(P)-[7 \mathrm{H}](\mathrm{b})$. For the saturated monolayer lattices, opposite tilt angles of cloverleaf clusters with respect to the adlattice are observed, indicated by red triangles in the unit cells, (c,d). (e) Model for the $M-[7] \mathrm{H}$ cloverleaf structure obtained from MMC. Minimal repulsion is achieved for certain relative azimuthal orientations.

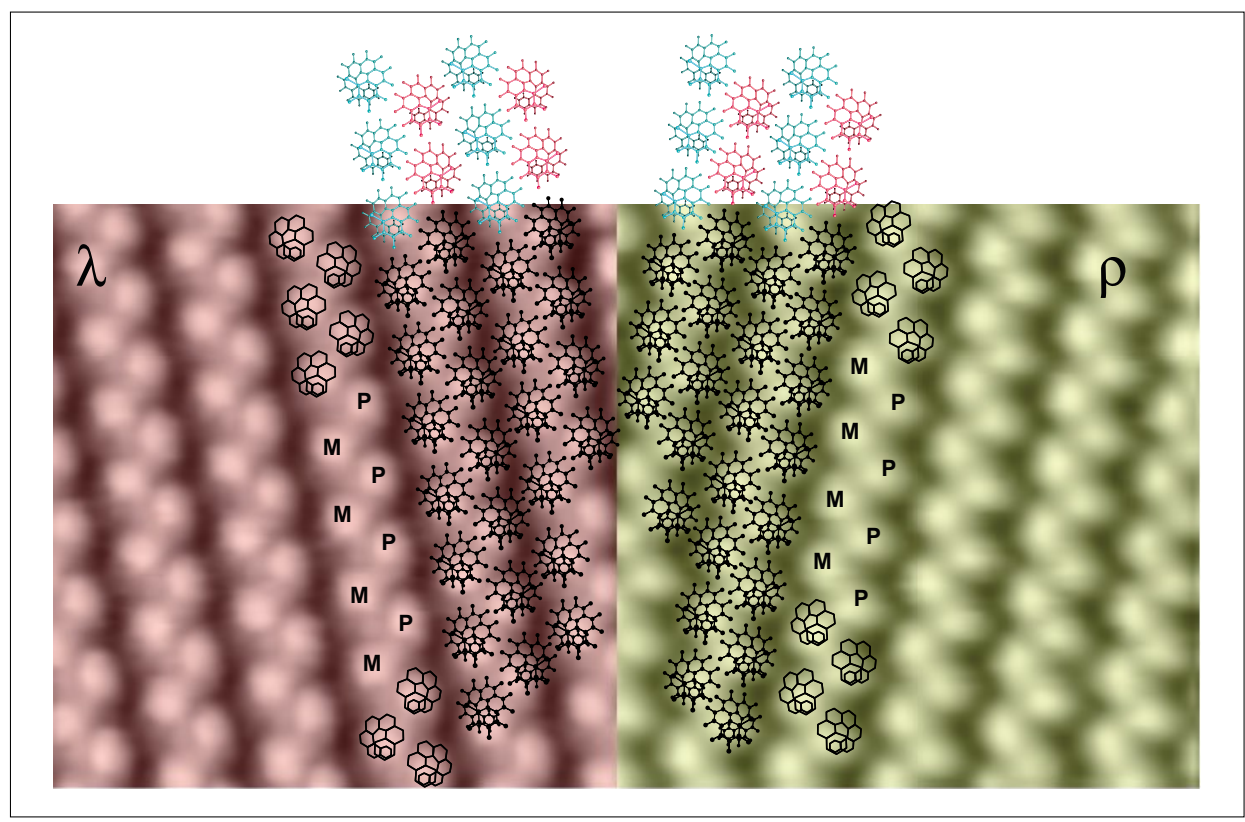

Fig. 2. Racemic [7] H on $\mathrm{Cu}(111)$. Superposition of the molecular orientation and STM images $(10 \mathrm{~nm} \times 10 \mathrm{~nm})$ of the mirror domains $(\lambda \& \rho)$ of the highest coverage structure. The observed enantiomorphism is achieved via alignment of heterochiral pairs in opposite chiral conformations.

interactions are expected. For lateral resolution of [7] $\mathrm{H}$ on $\mathrm{Cu}(111)$, this should lead to the same structures as observed for the isolated enantiomers. However, racemic [7] $\mathrm{H}$ forms a heterochiral lattice structure (Fig. 2). ${ }^{[13]}$ Nevertheless, mirror domains are observed via LEED and STM.[6b,13] Overall, three pairs of enantiomorphous structures, denoted as $\varepsilon / \delta, \lambda^{\prime} / \rho$ '. and $\lambda / \rho$, successively form with increasing coverage. ${ }^{[14]}$ All these adlattices have a common feature in that the molecules are aligned in zigzag double rows. The lattice structure of the $\lambda / \rho$ phase has been determined via high-resolution STM in combination with extended Hückel simulations as well as MMC (Fig. 2). The enantiomorphism is based on two possible relative alignments of the two enantiomers in a heterochiral pair on the surface. A glide plane along [IT0] interconverts the two enantiomers, but is not a symmetry element of a single domain lattice. It is noteworthy here that chiral expression at surfaces requires a minimum of mobility of the molecules. At nickel surfaces with their higher affinity to [7]H, for example, no dif- 
ferences for pure enantiomers and racemate have been observed. ${ }^{[15]}$

In the case of different local molecular adsorption geometries, lateral interactions vary substantially for a single compound with possible consequences for $2 \mathrm{D}$ enantiomeric resolution. This has been shown for racemic tartaric acid (TA) on $\mathrm{Cu}(110){ }^{[16]}$ Depending on temperature and/or coverage, TA forms different lattice structures on the surface. ${ }^{[17]}$ In particular, if one or both carboxylate groups react with the copper surface, bitartrate or monotartrate species will be present, respectively. For $(R, R)$-TA, bitartrate species have been identified only after thermal activation at lower coverage. ${ }^{[17]}$ With increasing coverage, however, newly adsorbed TA molecules hydrogenate the doubly deprotonated bitartrate species to monotartrate again. ${ }^{[17]}$ Besides this change in local adsorption geometry, an enantiomorphous $(90,12)$ bitartrate lattice undergoes a phase transition into a (4 0, 2 1) lattice. ${ }^{[18]}$ Interestingly, this lattice has only the substrate symmetry, i.e. no chirality is expressed at the supramolecular level. With further exposure to enantiopure TA, a slightly denser $(41,25)$ structure is formed. ${ }^{[17]}$ This structure breaks again the mirror symmetry of the underlying copper substrate, i.e. it is enantiomorphous. For racemic $\mathrm{TA}$, on the other hand, a $2 \mathrm{D}$ conglomerate is observed only for the bitartrate species, where homochiral $(90,12)$ and $(90,-12)$ domains coexist on the surface. ${ }^{[16]}$ The corresponding LEED pattern shows a superposition of both structures (Fig. 3). The racemate forms a $(40,21)$ monotartrate structure as well, but in contrast to the pure enantiomers, this is the monolayer saturation structure. This difference in achievable packing density for pure enantiomer and racemate is a strong indication that the two $(40,21)$ structures must be different. For a conglomerate of coexisting (4 0, 2 1) mirror domains, further exposure to racemic TA should lead - equivalent to the enantiopure case - to coexisting $(41, \pm 25)$ mirror domains. This difference is evidence for a heterochiral $(40,21)$ racemate lattice, not allowing additional inclusion of TA molecules. The lower thermal stability of the $(40,21)$ racemate structure with respect to the same structure of enantiopure TA supports this scenario. ${ }^{[19]}$ Monotartrate decomposes upon heating into carbon dioxide, water, carbon and hydrogen on $\mathrm{Cu}(110)$. The stability of monotartrate is substantially enhanced due to the close-packed monolayer structure, not allowing interaction of upper parts of the molecule with the surface in the first place. Consequently, the $\mathrm{CO}_{2}$ TPD signal, reflecting the decomposition reaction, shifts to higher temperatures with increasing coverage (Fig. 3). Moreover, this reaction is catalyzed by free surfaces sites. Since the decomposition, in turn, creates ac-

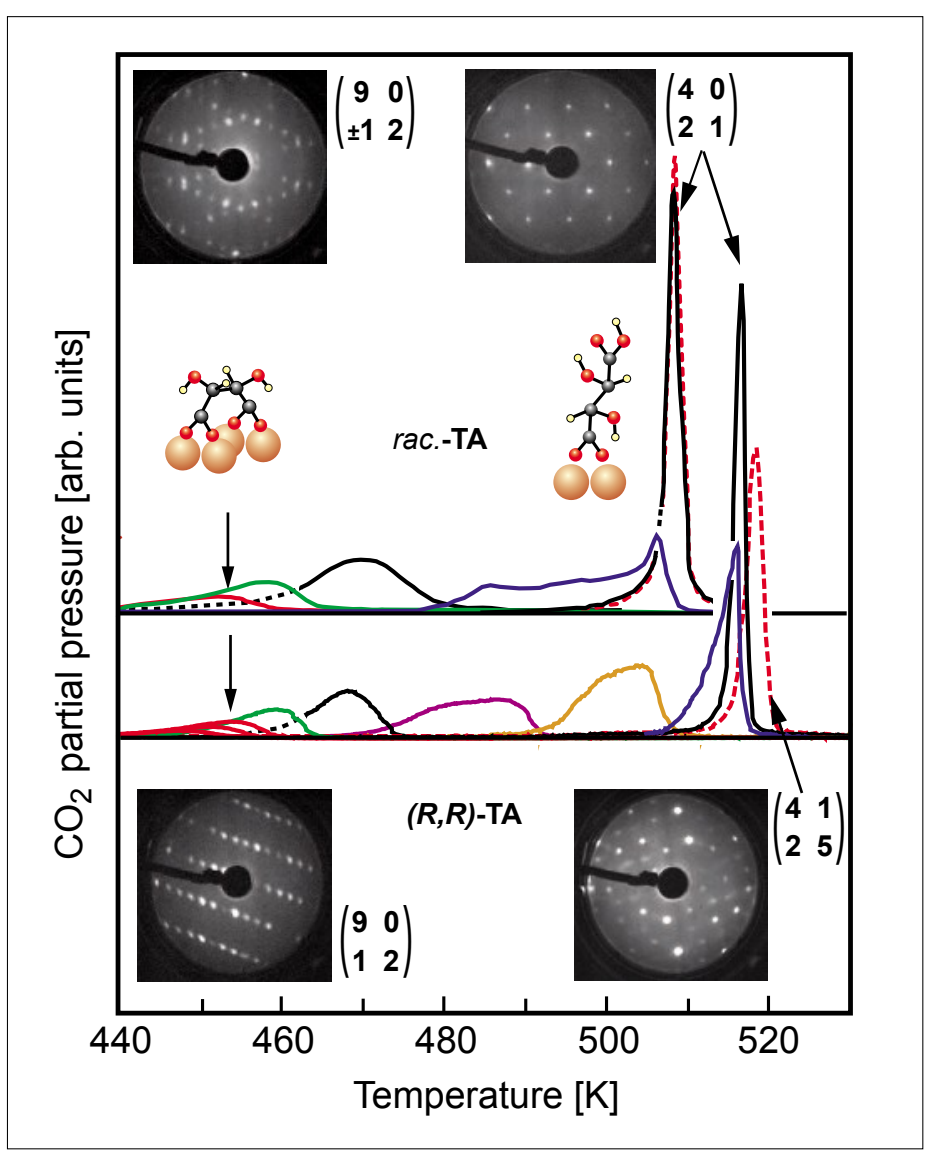

Fig. 3. LEED patterns and TPD curves for $(R, R)$-TA (bottom) and racemic TA (top). At $405 \mathrm{~K},(R, R)-\mathrm{TA}$ forms a $(90,12)$, a $(40,21)$ (not shown here) and a $(41,25)$ structure with increasing coverage. For the racemate a superposition of the mirror-related $(90,12)$ and $(90,-12)$ domains as well as a $(40,21)$ structure are observed. The arrows point at the TPD traces for the respective lattice structures. While the thermal stability of the bitartrate structures for racemate and pure enantiomers is identical, it is lower by $8 \mathrm{~K}$ for the racemate $(40,21)$ monotartrate structure compared to the $(R, R)-(40,21)$ monotartrate structure.

tive sites, the reaction is under autocatalytic control. Upon heating, this leads to complete decomposition in a very narrow temperature interval $(2 \mathrm{~K})$ once the reaction has started, because the gaseous decomposition products desorb instantaneously at decomposition temperature and a sharp pressure rise is observed. This is shown in Fig. 3 for $\mathrm{CO}_{2}$ as product. Interestingly, the decomposition temperature for the enantiopure $(40,21)$ structure exceeds the one for the same racemic structure by $8 \mathrm{~K}$. This higher stability is explained by a supramolecular chiral ensemble effect. The initial decomposition step requires a rearrangement of the upper part of the molecule in order to reach for the surface in a densely packed environment. The difference in handedness of adjacent molecules affects the extended H-bonding network of enantiopure and racemic lattices and has consequences for this initial 'unhinging' process. Hence, the lower thermal stability is due to more heterogeneous bonding and leads again to the conclusion that the racemate forms a heterochiral $(40,21)$ monotartrate lattice. Consequently, the coexisting homochiral
$(90, \pm 12)$ bitartrate domains show the same thermal stability as the $(R, R)-(90,12)$ bitartrate structure (Fig. 3).

\subsection{Adsorption-induced Chirality}

Achiral molecules can become chiral upon adsorption, either due to asymmetric distortion of the molecular frame or just because of an adsorbate complex devoid of mirror symmetry. ${ }^{[9]}$ However, all adsorption-induced chirality processes have in common that both enantiomers will be created as long as no further bias for single handedness is present. At a global level, the surface remains achiral, but at a local level, spontaneous symmetry breaking is a common phenomenon. As for intrinsically chiral molecules, the induced local chirality of adsorbed molecules is often transferred into the adlattice, which is then aligned in an oblique angle with respect to the substrate lattice. Again, these enantiomorphous structures are then easily observed via LEED. We note that mirror domains may be created with no chiral adsorbate complex involved, because the optimal packing arrangement breaks the symmetry 
of the underlying substrate lattice. ${ }^{[9,20]}$ For $(R, R)$ - and $(S, S)$-bitartrate a zigzag distortion was determined experimentally.[21] Density functional theory (DFT) calculations, however, also predict a chiral zigzag conformation after deprotonation of both carboxyl groups for achiral $(R, S)$-TA (meso-tartaric acid) and succinic acid (SU) on $\mathrm{Cu}(110)$. $^{[22]}$ Experimental observations of long-range enantiomorphous patterns in connection with reasonable molecular structure considerations indeed suggest a chiral geometry for $(R, S)$-bitartrate and bisuccinate on $\mathrm{Cu}(110) .{ }^{[23,24]}$ Because the probability of generating both enantiomorphous lattices is identical, two-dimensional conglomerates are formed, i.e. all molecules in a single domain of the adlattice have the same chirality. As in the case of racemic bitartrate, both mirror structures are observed in the LEED pattern, provided that the size of the probing electron beam is larger than the average domain size.

\subsection{Amplification of Chirality}

As shown above, adsorption-induced chirality creates a bistable system. Additional chiral bias, however, may suppress one state if the barrier of interconversion is not too high. The coadsorption of chiral molecules into such racemic layers is an efficient way to induce further asymmetrization towards single handedness. A small amount of a chiral impurity can be sufficient for induction of homochirality on the entire surface! SU on $\mathrm{Cu}(110)$, for example, switches from one handedness to the other in its bisuccinate phase when the temperature is raised above $500 \mathrm{~K}$. Cooling down such layer after doping with one TA enantiomer completely suppresses the formation of one mirror domain and installs global homochirality. ${ }^{[25]}$ This process is illustrated in Fig. 4. Consequently, the opposite TA-enantiomer suppresses the opposite SU enantiomorph, which is easily detectable in LEED (Fig. 4). Only 2\% of chiral dopant is necessary to install global homochirality. Smaller amounts of dopant lead to a lower intensity of the diffraction spots of the less favored enantiomorphous lattice. A similar effect - coined as the sergeant-and-soldiers principle - has been observed for helical polymers where a small concentration of chiral side chains induced single helicity. [26] Since hydrogen bonds between the bisuccinate molecules as a means of transferring chirality can safely be excluded, one must consider a substrate-mediated mechanism. That is, a chiral footprint in the surface acts as a chiral bias and suppresses opposite handedness in the adjacent adsorbate complex. This explains the amplification of the preferred handedness due to chiral doping: The TA sergeant has only one kind of a chiral footprint, forcing adjacent SU molecules into similar configuration. These,

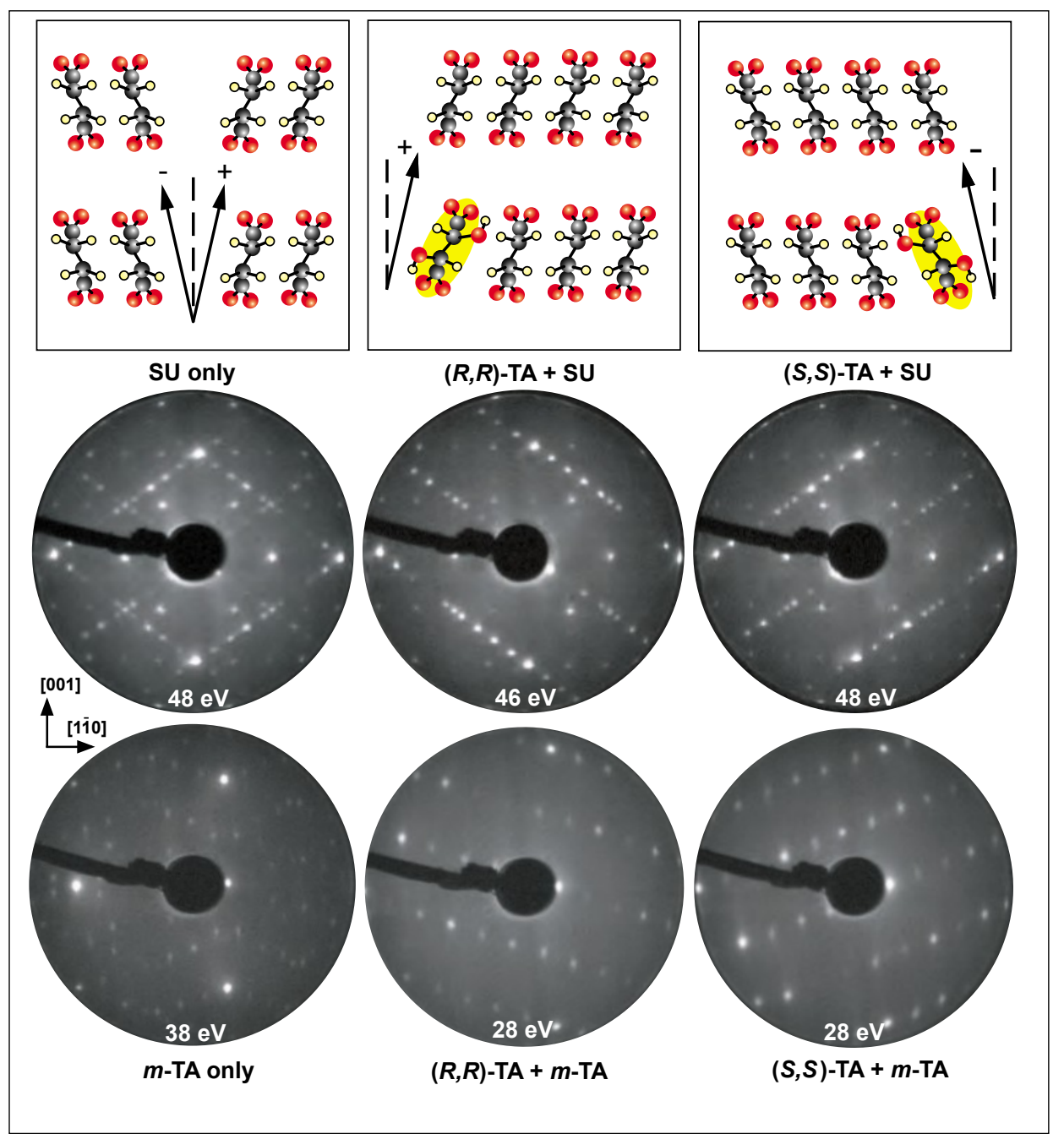

Fig. 4. Sergeant-and-soldiers principle at surfaces revealed by LEED: Prochiral SU and $(R, S)-T A$ form conglomerates at the surface and both mirror domains are observed as superposition in the electron diffraction experiment (first column). Doping with chiral TA provides the chiral bias inducing homochirality in the entire $2 \mathrm{D}$ crystal. Electron beam energies and the $\mathrm{Cu}(110)$ lattice orientation are indicated.

in turn, force their SU neighbors into the same configuration for energetic reasons. Since achiral $(R, S)$-TA becomes chiral on $\mathrm{Cu}(110)$ as well, this type of homochirality induction can be likewise observed after $(S, S)$ - or $(R, R)$-TA doping (Fig. 4).[27]

In contrast to the chiral doping mechanism, a small enantiomeric excess (ee) may also induce lattice homochirality. ${ }^{[28]}$ As mentioned above, the heterochiral pairs of [7] $\mathrm{H}$ on $\mathrm{Cu}(111)$ may exist in two enantiomorphous states due to two possible alignment on the $\mathrm{Cu}(111)$ surface. The chiral bias from a small $e e$ is sufficient in order to suppress formation of one unfavorable mirror domain and induces homochiral lattice order. ${ }^{[13]}$ Because of strong steric constraints, any $e e$ is expelled from the racemic enantiomorphous domains during crystallization. From the domain edges, however, the excess molecules have an influence on the relative alignment of the heterochiral pairs at the domain edge. (M)-[7]H excess favors formation of $\lambda$ domain pairs and $(P)-[7] \mathrm{H}$ excess favors the $\rho$ domain-pair-alignment. This chiral bias is then amplified by the cooperative interaction among heterochiral pairs, strongly favoring their equal alignment. Like helix reversals in a polymer chain, opposite alignment would create energetically unfavorable mirror domain boundaries. Even for the pure racemate, these boundaries are rarely observed on single $\mathrm{Cu}(111)$ terraces. Hence, spontaneous symmetry breaking is observed without chiral bias on a smaller scale, but the probability for $\lambda$ and $\rho$ domain decorated terraces is equal. At $e e= \pm 0.08$ the entire surface - although still close to racemic content - is already driven into a homochiral arrangement during 2D crystallization and only one of either domain is observed (Fig. 5). In contrast to the SU/TA-doped and $(R, S)$-TA/TA doped systems, where the molecular frame of the molecule is switched to its mirror configuration, interconversion between both structures requires only a change in relative position of both enantiomers of a heterochiral pair. The TA-doped systems are truly ho- 

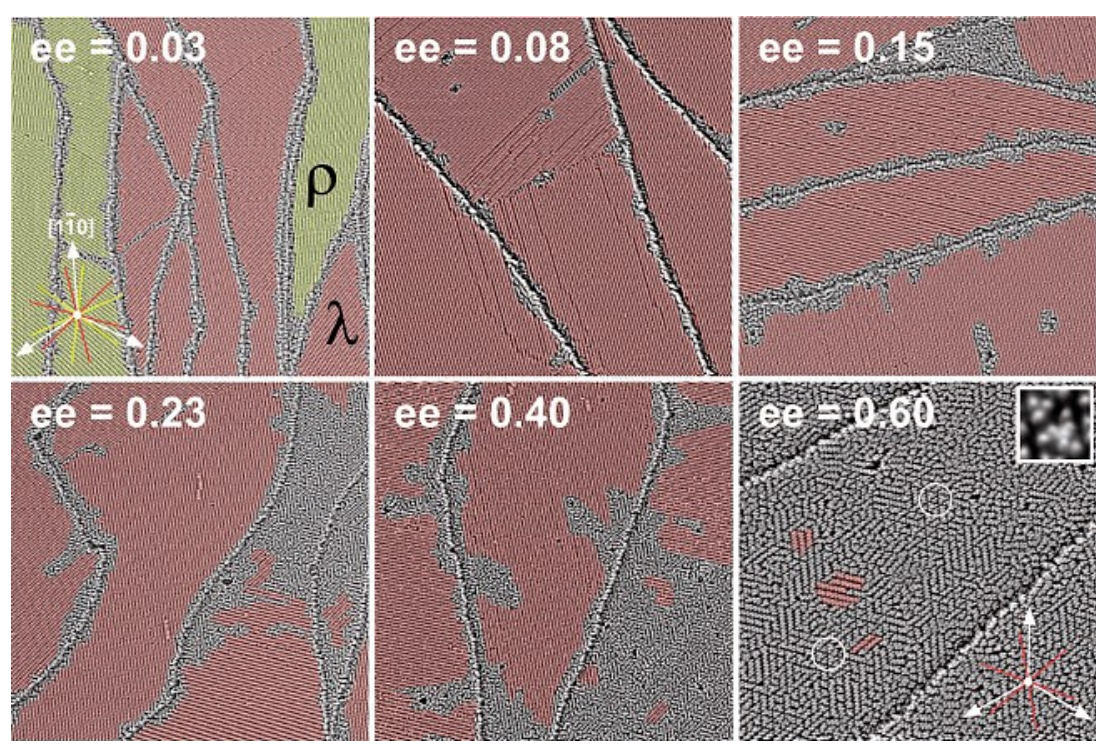

Fig. 5. STM images $(200 \mathrm{~nm} \times 200 \mathrm{~nm})$ of $[7] \mathrm{H}$ on $\mathrm{Cu}(111)$ with increasing enantiomeric excess. At $e e=0.08$ only a single mirror domain type $(\lambda)$ can exist on the surface. The excess (grey area) is expelled from the heterochiral domain, and with increasing ee, the excess area increases accordingly.

mochiral, i.e. all molecules have the same handedness. The ee-bias-amplified [7] $\mathrm{H} /$ $\mathrm{Cu}(111)$ system, however, is still of heterochiral composition, but possesses a lattice homochirality. In order to observe this eeeffect, lateral resolution of the enantiomers is not allowed.

\section{Summary}

In two-dimensional molecular systems the consequences of cooperativity are wellobserved via STM or surface diffraction methods. Long-range chiral motifs are formed after adsorption of chiral molecules or due to spontaneous symmetry breaking of prochiral molecules. Additional chiral bias due to chiral impurities (dopant) or small enantiomeric excess leads via cooperative amplification to homochirality in the $2 \mathrm{D}$ molecular lattice.

\section{Acknowledgement}

Support by the Schweizerischer Nationalfonds is gratefully acknowledged.

Received: February 15, 2008
[1] L. Pasteur, Ann. Chim. Phys. 1848, 24, 442. [2] J. D. Dunitz, Chem. Commun. 2003, 545.

[3] J. Jacques, A. Collet, S. H. Wilen, 'Enantiomers, Racemates and Resolutions', Krieger Publishing Company, Malabar, Florida, 1994

[4] C. P. Brock, W. B. Schweitzer, J. D Dunitz, J. Am. Chem. Soc. 1991, 113, 9811.

[5] J. S. Siegel, Nature 2001, 409, 777.

[6] a) A. Sudharkar, T. J. Katz, Tetrahedron Lett. 1986, 27, 2231; b) K.-H. Ernst, Y. Kuster, R. Fasel, M. Müller, U. Ellerbeck, Chirality 2001, 13, 675.

[7] T. Bürgi, A. Urakawa, B. Behzadi, K.-H. Ernst, A. Baiker, New J. Chem. 2004, 28, 332.

[8] R. Fasel, A. Cossy, K.-H. Ernst, F. Baumberger, T. Greber, J. Osterwalder, $J$. Chem. Phys. 2001, 115, 1020.

[9] K.-H. Ernst, Top. Curr. Chem. 2006, 265, 209.

[10] R. Fasel, M. Parschau, K.-H. Ernst, Angew. Chem., Int. Ed. 2003, 42, 5178; Angew. Chem. 2003, 115, 5336.

[11] S. M. Barlow, R. Raval, Surf. Sci. Rep. 2003, 50, 201

[12] W. Stocker, M. Schumacher, S. Graff, A. Thierry, J. C. Wittmann, B. Lotz, Macromolecules 1998, 31, 807.
[13] R. Fasel, M. Parschau, K.-H. Ernst, Nature 2006, 439, 449.

[14] M. Parschau, R. Fasel, K.-H. Ernst, Cryst. Growth \& Des. 2008, in print.

[15] a) K.-H. Ernst, Y. Kuster, R. Fasel, C. F. McFadden, U. Ellerbeck, Surf. Sci. 2003 , 530, 195; b) K.-H. Ernst, M. Neuber, M. Grunze, U. Ellerbeck, J. Am. Chem. Soc. 2001, 123, 493.

[16] S. Romer, B. Behzadi, R. Fasel, K.-H. Ernst, Chem. Eur. J. 2005, 11, 4149.

[17] M. Ortega Lorenzo, S. Haq, T. Bertrams, P. Murray, R. Raval, C. J. Baddeley, J. Phys. Chem. B 1999, 103, 10661.

[18] The expression in parentheses stands for the $2 \times 2$ transformation matrix connecting adsorbate lattice with substrate surface lattice.

[19] B. Behzadi, S. Romer, R. Fasel, K.-H. Ernst, J. Am. Chem. Soc. 2004, 126, 9176.

[20] M. Parschau, R. Fasel, K.-H. Ernst, O. Gröning, L. Brandenberger, R. Schillinger, T. Greber, A. Seitsonen, Y.-T. Wu, J. S. Siegel, Angew. Chem., Int. Ed. 2007, 46, 8258; Angew. Chem. 2007, 119, 8406.

[21] R. Fasel, J. Wider, C. Quitmann, K.-H. Ernst, T. Greber, Angew. Chem., Int. Ed. 2004, 43, 2853.

[22] L. A. M. M. Barbosa, P. Sautet, J. Am Chem. Soc. 2001, 123, 6639.

[23] V. Humblot, M. Ortega Lorenzo, C. J. Baddeley, S. Haq, R. Raval, J. Am. Chem. Soc. 2004, 126, 6460.

[24] M. Parschau, B. Behzadi, S. Romer, K.-H. Ernst, Surf. Interface Anal. 2006, 38, 1607

[25] M. Parschau, S. Romer, K.-H. Ernst, J. Am. Chem. Soc. 2004, 126, 15398.

[26] M. M. Green, M. P. Reidy, R. J. Johnson, G. Darling, D. J. O'Leary, G. Wilson, J. Am. Chem. Soc. 1989, 111, 6454.

[27] M. Parschau, T. Kampen, K.-H. Ernst, Chem. Phys. Lett. 2005, 407, 433.

[28] K.-H. Ernst, Curr. Opin. Coll. Interf. Sci. 2008, 13, 54. 